\title{
MMSE Based Greedy Antenna Selection Scheme for AF MIMO Relay Systems
}

\author{
Ming Ding, Shi Liu, Hanwen Luo, and Wen Chen, Member, IEEE
}

\begin{abstract}
We propose a greedy minimum mean squared error (MMSE)-based antenna selection algorithm for amplify-andforward (AF) multiple-input multiple-output (MIMO) relay systems. Assuming equal-power allocation across the multi-stream data, we derive a closed form expression for the mean squared error (MSE) resulted from adding each additional antenna pair. Based on this result, we iteratively select the antenna-pairs at the relay nodes to minimize the MSE. Simulation results show that our algorithm greatly outperforms the existing schemes.
\end{abstract}

Index Terms-AF, MIMO relay, antenna selection, MMSE.

\section{INTRODUCTION}

Multipe-Input multiple-output (MIMO) relay systems have been recognized to achieve a large diversity gain and a large spectrum efficiency [1]. Meanwhile, amplify-and-forward (AF) MIMO relay systems have drawn extensive attentions in the literature due to their simplicity and mathematical tractability [1]-[9]. In order to achieve the theoretical capacity shown in [1], [2], many advanced signal processing schemes have been proposed. The authors of [2] introduced the "doubly coherent" backward and forward matched filtering strategy. In [3], backward matched filtering and forward zero-forcing (ZF) precoded transmission was proposed. In [4], singular value decomposition (SVD) for backward channel and ZF dirty paper coding at the source node was investigated.

However, most prior work entails that relay nodes should be equipped more antennas than the source or destination node so that the relays can perform backward interference-suppressing reception and forward precoding function. Unfortunately, this is not a realistic assumption since in practice, relays basically serve as a low-cost and low-complexity means to extend the coverage and enhance the spectrum efficiency for cell edge users [5]. Therefore, in the practical cases that advanced signal processing cannot be relegated to the relay nodes, relay/antenna selection becomes an attractive option.

A few antenna selection techniques designed for single antenna/stream relay networks [6], [7] have been reported recently. Many on-going works regarding antenna selection for multi-stream AF MIMO relay systems unfold more interesting

Copyright (c) 2008 IEEE. Personal use of this material is permitted. However, permission to use this material for any other purposes must be obtained from the IEEE by sending a request to pubs-permissions@ieee.org

The authors are with the Department of Electronics Engineering, Shanghai Jiaotong University, China (E-mail: \{dm2007; liushi_9851; hwluo; wenchen\}@sjtu.edu.cn). Wen Chen is also with SEU National Key Lab for mobile communications.

The authors would like to thank Dr. Sayantan Choudhury from Sharp Lab of America and several anonymous reviewers for their careful review on earlier versions of the manuscript. This work is supported by NSFC 60972031, SEU SKL for Mobile communications W200907. thoughts. In [8], a heuristic relay/antenna selection criterion based on harmonic mean of dual-hop sub-channel gains was proposed. In [9], the authors proposed an iterative antenna selection scheme based on semi-orthogonality among the selected antenna pairs. However, both [8] and [9] overlooked the noise at the relay nodes, which might be too ideal in practice. Moreover, instead of using heuristic methods, it would be better to develop an antenna selection scheme based on more concrete criteria in closed forms, such as capacity maximization or minimum mean squared error (MMSE). Thus, in this letter we propose a MMSE-based greedy antenna selection algorithm for AF MIMO relay systems. Simulation results validate the superiority of our scheme compared to those in [8] and [9], with the gain being more pronounced when the noise at the relay nodes is relatively large.

In this letter, $(\cdot)^{\mathrm{T}},(\cdot)^{\mathrm{H}},(\cdot)^{-1}, \operatorname{det}(\cdot)$, and $\operatorname{tr}(\cdot)$ stand for the transpose, conjugate transpose, inverse, determinant and trace of a matrix, respectively. $\varepsilon(\cdot)$ is the expectation of a random variable. $|\mathbf{a}|$ denotes the Euclidean norm of a vector $\mathbf{a} .(\mathbf{A})_{i, i}$ is the $i$ th diagonal entry of the matrix $\mathbf{A}$. $\mathbf{I}_{N}$ stands for an $N \times$ $N$ identity matrix. Finally, $\mathrm{C}_{K}^{l}$ counts the events of selecting $l$ elements from a homogeneous set containing $K$ elements.

\section{System Model}

The dual-hop MIMO relay system considered in this letter is illustrated by Fig. 1, where the source node $(\mathcal{S})$, the destination node $(\mathcal{D})$ and each relay node $\mathcal{R}_{k}(k=1, \cdots, K)$ are equipped with $N_{\mathrm{s}}, N_{\mathrm{d}}$, and $N_{\mathrm{r}}$ antennas, respectively. $\mathbf{H}_{k} \in \mathbb{C}^{N_{\mathrm{r}} \times N_{\mathrm{s}}}$ and $\mathbf{G}_{k} \in \mathbb{C}^{N_{\mathrm{d}} \times N_{\mathrm{r}}}$ denote the backward channels $\left(\mathcal{S} \rightarrow \mathcal{R}_{k}\right)$ and the forward channels $\left(\mathcal{R}_{k} \rightarrow \mathcal{D}\right)$ respectively. All the channels are modeled as block-wise flat fading. Throughout the letter, we only focus on Half Time Division Duplex (HTDD) relaying with AF protocol, i.e., the transmission time interval is divided into 2 time slots. The first and the second time slot of which are assigned to the backward and the forward transmission respectively.

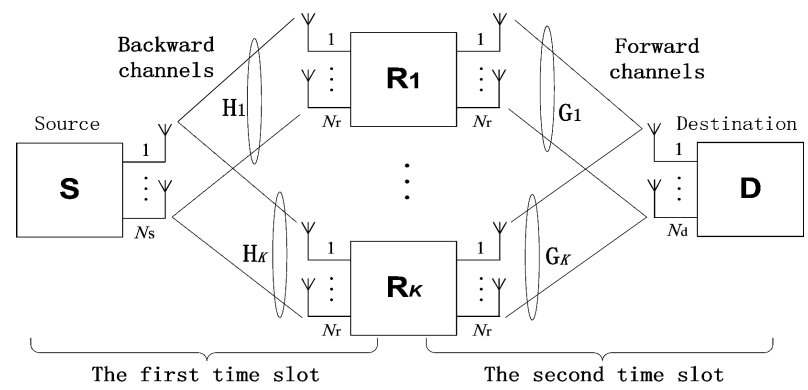

Fig. 1. Schematic model of an AF MIMO relay system. 
Taking the issues of practical implementation into consideration, we here assume that each MIMO relay node is equipped with only one power amplifier (PA) so that only one antenna pair can be activated on each relay node for the HTDD based transmission. We will select $L$ antenna pairs in the MIMO relay network to bridge the communication from $\mathcal{S}$ to $\mathcal{D}$. Let the $L$ antenna pairs be associated with a relay set $\Gamma=\{r(l) \mid l=1, \cdots, L\}$, which contains distinct elements due to the single-PA assumption. It should be pointed out that the single-PA restriction can be easily lifted to multi-PA by using the eigenmode construction [9], i.e., SVD operations are performed for $\mathbf{H}_{k}, \mathbf{G}_{k}$, resulting in eigenmode-based definitions for the equivalent backward and forward channels. Here we consider a case $K \geq N_{\mathrm{s}}, N_{\mathrm{d}}$. Thereby, the multiplexing gain of the interested system is limited by $M=\min \left(N_{\mathrm{s}}, N_{\mathrm{d}}\right)$. If full multiplexing gain is achieved between $\mathcal{S}$ and $\mathcal{D}, L$ should be no less than $M$ in order to make the equivalent twohop relay channel well-conditioned. In [8] and [9], $L$ is set to $M$ due to the dimensional condition of the orthogonal subchannels, whereas in our scheme $L$ is encouraged to be larger than $M$ so that more diversity could be exploited to benefit the MSE performance. For simplicity, we assume $N_{\mathrm{d}} \geq N_{\mathrm{s}}=M$ in the sequel without loss of generality.

The $L$ antenna pairs generate a compound backward channel $\mathbf{H}=\left[\mathbf{h}_{(b(1), r(1))}^{\mathrm{T}}, \cdots, \mathbf{h}_{(b(l), r(l))}^{\mathrm{T}}, \cdots, \mathbf{h}_{(b(L), r(L))}^{\mathrm{T}}\right]^{\mathrm{T}}$, where $\mathbf{h}_{(b(l), r(l))}$ is the $1 \times N_{\mathrm{s}}$ channel vector of $\mathcal{S}$ to the $l$ th selected backward antenna $b(l)$ on relay $r(l)$. During the first time slot, let the received signals at the $L$ backward antennas be stacked into a vector $\mathbf{y}=\left[y_{1}, y_{2}, \cdots, y_{L}\right]^{\mathrm{T}}$, which is given by

$$
\mathbf{y}=\mathbf{H} \mathbf{x}+\mathbf{n}_{\mathrm{r}}
$$

where $\mathbf{x} \in \mathbb{C}^{N_{\mathrm{s}}}$ is the transmit signal vector, and $\mathbf{n}_{\mathrm{r}} \in \mathbb{C}^{L}$ denotes the white zero-mean circularly symmetric complex Gaussian (ZMCSCG) noise vector with covariance matrix $\mathbf{I}_{L}$. We further denote by $P_{\mathrm{s}}$ the total transmit power available at $\mathcal{S}$ and put constraint on $\mathrm{x}$ as

$$
\varepsilon\left\{\operatorname{tr}\left(\mathbf{x x}^{\mathrm{H}}\right)\right\}=\operatorname{tr}\left(\varepsilon\left\{\mathbf{x x}^{\mathrm{H}}\right\}\right) \leq P_{\mathrm{s}} .
$$

To derive tractable solutions, we assume equal power allocation across the transmitted data streams at $\mathcal{S}$ with full power. Hence, the covariance matrix of $\mathbf{x}$ is $\sigma_{x}^{2} \mathbf{I}_{N_{\mathrm{s}}}$, and $\sigma_{x}^{2}=P_{\mathrm{s}} / N_{\mathrm{s}}$.

In the second time slot, relays in $\Gamma$ amplify and forward the received signal $\mathbf{y}$ to $\mathcal{D}$. We assume that no direct link is available from $\mathcal{S}$ to $\mathcal{D}$ due to long-distance pathloss. The AF relays' amplifying function can be represented by a diagonal matrix $\mathbf{W} \in \mathbb{C}^{L}$, whose diagonal scalar entry $w_{l}$ is the gain associated with the $l$ th selected forward relay antenna $(f(l), r(l))$ (antenna $f(l)$ of relay $r(l)$ ). It should be noted that previously defined $b(l)$ is not necessary to be the same as $f(l)$ in the MIMO relay node. But they have to belong to the same relay $r(l)$ because no cooperation is operated among different relays. From (1), the amplified signal is written as

$$
\mathbf{t}=\mathbf{W y}=\mathbf{W H x}+\mathbf{W n}_{\mathbf{r}} .
$$

Usually, each relay node has an independent power supply, the local power of which is bounded by $P_{\text {loc }}$ shown as

$$
\varepsilon\left\{\operatorname{tr}\left(\mathbf{t t}^{\mathrm{H}}\right)_{l, l}\right\}=\left(\mathbf{W}^{2}\left(\sigma_{x}^{2} \mathbf{H} \mathbf{H}^{\mathrm{H}}+\mathbf{I}_{L}\right)\right)_{l, l} \leq P_{\mathrm{loc}} .
$$

Assume full power in (4). Then $w_{l}$ can be represented by

$$
w_{l}=\sqrt{P_{\mathrm{loc}} /\left(\sigma_{x}^{2}\left|\mathbf{h}_{(b(l), r(l))}\right|^{2}+1\right)} .
$$

Under the condition of perfect synchronization in the relay network, signal arriving at $\mathcal{D}$ is given by

$$
\mathbf{z}=\mathbf{G t}+\mathbf{n}_{\mathrm{d}}=\mathbf{G W H x}+\mathbf{G W \mathbf { n } _ { \mathrm { r } }}+\mathbf{n}_{\mathrm{d}},
$$

where the noise term $\mathbf{n}_{\mathrm{d}} \in \mathbb{C}^{N_{\mathrm{d}}}$ stands for the ZMCSCG vector at $\mathcal{D}$ with identity covariance matrix. $\mathbf{G} \in$ $\mathbb{C}^{N_{\mathrm{d}} \times L}$ denotes the compound forward channel written as $\mathbf{G}=\left[\mathbf{g}_{(f(1), r(1))}, \cdots, \mathbf{g}_{(f(l), r(l))}, \cdots, \mathbf{g}_{(f(L), r(L))}\right]$. By denoting equivalent channel $\mathbf{H}_{\mathrm{eq}}=\mathbf{G W H}$ and colored noise term $\mathbf{n}_{\mathrm{eq}}=\mathbf{G W} \mathbf{n}_{\mathrm{r}}+\mathbf{n}_{\mathrm{d}}$, (6) can be further reduced to $\mathbf{z}=\mathbf{H}_{\mathrm{eq}} \mathbf{x}+\mathbf{n}_{\mathrm{eq}}$. According to [10], the MSE of symbol estimation will achieve its minimum value when Wiener filter is employed. The corresponding MSE is presented by

$$
\begin{aligned}
Q & =\sigma_{x}^{2} \operatorname{tr}\left\{\left(\mathbf{I}_{N_{\mathrm{d}}}+\sigma_{x}^{2} \mathbf{H}_{\mathrm{eq}} \mathbf{H}_{\mathrm{eq}}^{\mathrm{H}} \mathbf{\Phi}^{-1}\right)^{-1}\right\}+\sigma_{x}^{2}\left(N_{\mathrm{s}}-N_{\mathrm{d}}\right) \\
& =\sigma_{x}^{2} \operatorname{tr}\left\{\mathbf{\Phi}\left(\mathbf{\Phi}+\sigma_{x}^{2} \mathbf{H}_{\mathrm{eq}} \mathbf{H}_{\mathrm{eq}}^{\mathrm{H}}\right)^{-1}\right\}+\beta,
\end{aligned}
$$

where $\boldsymbol{\Phi}=\varepsilon\left\{\mathbf{n}_{\mathrm{eq}} \mathbf{n}_{\mathrm{eq}}^{\mathrm{H}}\right\}=\mathbf{G W}(\mathbf{G W})^{\mathrm{H}}+\mathbf{I}_{N_{\mathrm{d}}}$ is the covariance matrix for $\mathbf{n}_{\text {eq }}$. Since $\beta=\sigma_{x}^{2}\left(N_{\mathrm{s}}-N_{\mathrm{d}}\right)$ is just a constant that is free from the minimization of $Q$, we will omit $\beta$ hereafter.

\section{The Proposed Antenna Selection Algorithm}

Since (7) is a closed-form expression to evaluate the MSE of the system, we can perform an exhaustive search to minimize $Q$ to obtain the optimal antenna pair set. However, such a brute-force search is quite infeasible considering the required trials could be as large as $\sum_{l=M}^{K} \mathrm{C}_{K}^{l}\left(N_{\mathrm{r}}^{2}\right)^{l}$, where $N_{\mathrm{r}}^{2}$ refers to the number of candidate antenna pairs at each relay node. In a modest case where $N_{\mathrm{s}}=N_{\mathrm{d}}=4, N_{\mathrm{r}}=2$, and $K=8$, the exhaustive search would involve approximately $3.9 \times 10^{5}$ trials requiring the inversion of a matrix of size $4 \times 4$ in each trial shown in (7). Moreover, that figure would soon rocket to nearly 10 million if $K=10$. Therefore, instead of approaching (7) directly, we investigate how the MSE is affected when one more antenna pair is chosen so as to find a way to optimize the system asymptotically.

Denote $\mathbf{H}_{l}=\left[\mathbf{h}_{(b(1), r(1))}^{\mathrm{T}}, \cdots, \mathbf{h}_{(b(l), r(l))}^{\mathrm{T}}\right]^{\mathrm{T}}$ as the already selected backward channel, then the diagonal entries of $\mathbf{W}_{l}$ can be obtained from (5). Next we turn to the $(l+1)$ th antenna pair. For every un-selected candidate backward channel $\mathbf{h}_{m, k}$ of $\mathcal{S}$ to the $m$ th antenna of the $k$ th relay, we calculate the associated relay gain $w_{m, k}$ according to (5) as

$$
w_{m, k}=\sqrt{P_{\mathrm{loc}} /\left(\sigma_{x}^{2}\left|\mathbf{h}_{m, k}\right|^{2}+1\right)} .
$$

Suppose that $\mathbf{G}_{l}=\left[\mathbf{g}_{(f(1), r(1))}, \cdots, \mathbf{g}_{(f(l), r(l))}\right]$ is the selected partial forward channel. Let $\mathbf{g}_{n, k}$ be the $N_{\mathrm{d}} \times 1$ channel 
vector characterizing the forward link from the $n$th antenna of the $k$ th relay to $\mathcal{D}$. For simplicity, we use the notation $(m, n, k)$ to represent the candidate antenna pair $(m, n)$ on the $k$ th relay. Further denote

$$
\begin{aligned}
& \boldsymbol{\Phi}_{l}=\mathbf{I}_{N_{\mathrm{d}}}+\mathbf{G}_{l} \mathbf{W}_{l}^{2} \mathbf{G}_{l}^{\mathrm{H}}, \\
& \mathbf{A}_{l}=\mathbf{\Phi}_{l}+\sigma_{x}^{2}\left(\mathbf{G}_{l} \mathbf{W}_{l} \mathbf{H}_{l}\right)\left(\mathbf{G}_{l} \mathbf{W}_{l} \mathbf{H}_{l}\right)^{\mathrm{H}}, \\
& \mathbf{F}_{m, n, k}=\mathbf{G}_{l} \mathbf{W}_{l} \mathbf{H}_{l}+w_{m, k} \mathbf{g}_{n, k} \mathbf{h}_{m, k}, \\
& \mathbf{u}_{m, n, k}=\sigma_{x}^{2}\left(\mathbf{G}_{l} \mathbf{W}_{l} \mathbf{H}_{l}\right) w_{m, k} \mathbf{h}_{m, k}^{\mathrm{H}}+w_{m, k}^{2} \mathbf{g}_{n, k}, \\
& \boldsymbol{v}_{m, n, k}=\sigma_{x}^{2} \mathbf{F}_{m, n, k} w_{m, k} \mathbf{h}_{m, k}^{\mathrm{H}} .
\end{aligned}
$$

Our main result is summarized in the following proposition.

Proposition 1: The MSE resulted from the $(l+1)$ th additional sub-channels $\mathbf{h}_{m, k}, \mathbf{g}_{n, k}$ with respect to the antenna pair $(m, n, k)$ is

$$
Q_{l+1}^{(m, n, k)}=\sigma_{x}^{2} \operatorname{tr}\left\{\left(\mathbf{\Phi}_{l}+w_{m, k}^{2} \mathbf{g}_{n, k} \mathbf{g}_{n, k}^{\mathrm{H}}\right) \mathbf{C}_{(m, n, k)}^{-1}\right\},
$$

where $\mathbf{C}_{m, n, k}^{-1}=\mathbf{B}_{m, n, k}^{-1}-\frac{\mathbf{B}_{m, n, k}^{-1} \mathbf{g}_{n, k} \boldsymbol{v}_{m, n, k}^{\mathrm{H}} \mathbf{B}_{m, n, k}^{-1}}{1+\boldsymbol{v}_{m, n, k}^{\mathrm{H}} \mathbf{B}_{m, n, k}^{-1} \mathbf{g}_{n, k}}$ and $\mathbf{B}_{m, n, k}^{-1}=\mathbf{A}_{l}^{-1}-\frac{\mathbf{A}_{l}^{-1} \mathbf{u}_{m, n, k} \mathbf{g}_{n, k}^{\mathrm{H}} \mathbf{A}_{l}^{-1}}{1+\mathbf{g}_{n, k}^{\mathrm{H}} \mathbf{A}_{l}^{-1} \mathbf{u}_{m, n, k}}$.

Poof 1: According to (7) and by some mathematical manipulations, the MSE (with $\beta$ omitted) with $\mathbf{h}_{m, k}$ and $\mathbf{g}_{n, k}$ added to $\mathbf{H}_{l}$ and $\mathbf{G}_{l}$ can be represented as

$$
\begin{aligned}
& Q_{l+1}^{(m, n, k)} \\
& =\sigma_{x}^{2} \operatorname{tr}\left\{\begin{array}{c}
\left(\mathbf{\Phi}_{l}+w_{m, k}^{2} \mathbf{g}_{n, k} \mathbf{g}_{n, k}^{\mathrm{H}}\right) \\
\times\left(\mathbf{\Phi}_{l}+w_{m, k}^{2} \mathbf{g}_{n, k} \mathbf{g}_{n, k}^{\mathrm{H}}+\sigma_{x}^{2} \mathbf{F}_{m, n, k} \mathbf{F}_{m, n, k}^{\mathrm{H}}\right)^{-1}
\end{array}\right\} \\
& =\sigma_{x}^{2} \operatorname{tr}\left\{\begin{array}{c}
\left(\mathbf{\Phi}_{l}+w_{m, k}^{2} \mathbf{g}_{n, k} \mathbf{g}_{n, k}^{\mathrm{H}}\right) \\
\left.\times\left(\mathbf{A}_{l}+\mathbf{u}_{m, n, k} \mathbf{g}_{n, k}^{\mathrm{H}}+\mathbf{g}_{n, k} \boldsymbol{v}_{m, n, k}^{\mathrm{H}}\right)^{-1}\right\} .
\end{array}\right.
\end{aligned}
$$

By invoking the matrix inversion lemma [11], that is

$$
\left(\mathbf{A}+\mathbf{X} \mathbf{Y}^{\mathrm{H}}\right)^{-1}=\mathbf{A}^{-1}-\mathbf{A}^{-1} \mathbf{X}\left(\mathbf{I}+\mathbf{Y}^{\mathrm{H}} \mathbf{A}^{-1} \mathbf{X}\right)^{-1} \mathbf{Y}^{\mathrm{H}} \mathbf{A}^{-1},
$$

where $\mathbf{A} \in \mathbb{C}^{a \times a}$ and $\mathbf{X}, \mathbf{Y} \in \mathbb{C}^{a \times b}$. We can evaluate (10) by means of a two-step recursion. Firstly, we denote $\mathbf{B}_{m, n, k}=$ $\mathbf{A}_{l}+\mathbf{u}_{m, n, k} \mathbf{g}_{n, k}^{\mathrm{H}}$, and calculate $\mathbf{B}_{m, n, k}^{-1}$ according to (11) as

$$
\mathbf{B}_{m, n, k}^{-1}=\mathbf{A}_{l}^{-1}-\frac{\mathbf{A}_{l}^{-1} \mathbf{u}_{m, n, k} \mathbf{g}_{n, k}^{\mathrm{H}} \mathbf{A}_{l}^{-1}}{1+\mathbf{g}_{n, k}^{\mathrm{H}} \mathbf{A}_{l}^{-1} \mathbf{u}_{m, n, k}} .
$$

Then let $\mathbf{C}_{m, n, k}=\mathbf{B}_{m, n, k}+\mathbf{g}_{n, k} \boldsymbol{v}_{m, n, k}^{\mathrm{H}}$. We have

$$
\mathbf{C}_{m, n, k}^{-1}=\mathbf{B}_{m, n, k}^{-1}-\frac{\mathbf{B}_{m, n, k}^{-1} \mathbf{g}_{n, k} \boldsymbol{v}_{m, n, k}^{\mathrm{H}} \mathbf{B}_{m, n, k}^{-1}}{1+\boldsymbol{v}_{m, n, k}^{\mathrm{H}} \mathbf{B}_{m, n, k}^{-1} \mathbf{g}_{n, k}} .
$$

The proof is completed by substituting (13) into (10). As shown in (9), $Q_{l+1}^{(m, n, k)}$ can be evaluated efficiently since $\mathbf{A}_{l}^{-1}$ is free from $(m, n, k)$ and only computed once for each l. Other computations involved in (9) are no more than several vector/matrix multiplications. Based on (9), we can iteratively activate more antenna pairs as long as the corresponding MSE keeps decreasing. Although this approach will not guarantee a global optimal solution as the exhaustive search, it has the potential to find a good local optimal solution because of three facts: (i) local optimality can be reflected in the nonincreasing MSE based searching; (ii) the noise terms have been correctly incorporated into (9); (iii) $L$ can be as large as $K$ to exploit the diversity gain of the network. Hence, we

\section{Algorithm 1 Greedy MSE Minimization (GMM)}

1) Initialization: Set $l=0, \mathbf{H}_{l}, \mathbf{G}_{l}, \mathbf{W}_{l}=\phi, \mathbf{A}_{l}=\mathbf{\Phi}_{l}=$ $\mathbf{I}_{N_{\mathrm{d}}}$, previous_MSE $=+\infty$; Let $\Omega$ be the candidate antenna pair set containing all $K N_{\mathrm{r}}^{2}$ pairs $(m, n, k)$.

2) Iterative loop: Compute $\mathbf{W}_{l}, \boldsymbol{\Phi}_{l}, \mathbf{A}_{l}$ and $\mathbf{A}_{l}^{-1}$;

For each antenna pair $(m, n, k)$ in $\Omega$, obtain $w_{m, k}$ according to (8). Then evaluate (9) to get $Q_{l+1}^{(m, n, k)}$.

$3)$ Select the $(l+1)$ th antenna pair by:

$$
(b(j), f(j), r(j))_{j=l+1}=\underset{(m, n, k)}{\arg \min }\left\{Q_{l+1}^{(m, n, k)}\right\} .
$$

4) If $\min \left\{Q_{l+1}^{(m, n, k)}\right\}<$ previous_MSE, then previous_MSE $=\min \left\{Q_{l+1}^{(m, n, k)}\right\}$;

Set $l=l+1$; Eliminate the $N_{\mathrm{r}}^{2}$ antenna pairs associated with the relay $r(l)$ from $\Omega$; Update $\mathbf{H}_{l}=$ $\left[\mathbf{H}_{l-1}^{\mathrm{T}}, \mathbf{h}_{(b(l), r(l))}^{\mathrm{T}}\right]^{\mathrm{T}}, \mathbf{G}_{l}=\left[\mathbf{G}_{l-1}, \mathbf{g}_{(f(l), r(l))}\right], \mathbf{W}_{l}=$ $\left[\begin{array}{cc}\mathbf{W}_{l-1} & 0 \\ 0 & w_{b(l), r(l)}\end{array}\right]$; Go to step 2 .

Else, terminate with $\mathbf{H}_{l}$ and $\mathbf{G}_{l}$ as the selected backward channel and forward channel.

propose a MMSE based greedy antenna selection algorithm summarized as follows.

\section{Simulation Results And Discussions}

A distributed orthogonal relay selection (DORS) algorithm and a semi-orthogonization (S-O) algorithm have been proposed in [8] and [9] respectively. The DORS algorithm selects the antenna pair to maximize the harmonic mean of dual-hop sub-channel gains achieved by $\mathbf{h}_{m, k}$ and $\mathbf{g}_{n, k}$, while in the S$\mathrm{O}$ algorithm the authors maximize the sum of the projection angles among the sub-channels of $\mathbf{H}_{l}$ and $\mathbf{G}_{l}$.

In our simulations, we adopt a realistic antenna setup as $N_{\mathrm{s}}=N_{\mathrm{d}}=M=4, N_{\mathrm{r}}=2$. The channels are assumed to be uncorrelated Rayleigh fading, which are modeled as i.i.d. ZMCSCG random variables with unit covariance. Furthermore, we denote the receive SNR at the relay nodes as $S N R_{1}=P_{\mathrm{s}}$ (with the noise power be normalized to 1 ). $P_{\text {loc }}$ is set to 5 $\mathrm{dB}$ above the noise power and 10000 Monte Carlo runs are conducted for each relay deployment.

Firstly, we make a brief complexity comparison among the aforementioned schemes. The exhaustive search scheme is nearly impossible to be analyzed when $K>8$, which is verified by our simulation efforts. The DORS and S-O algorithms stop the antenna selection procedure when $l=M$,

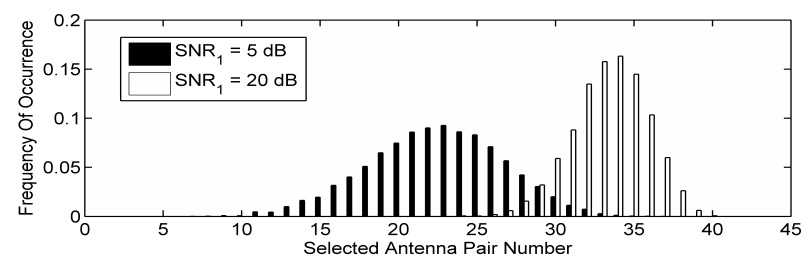

Fig. 2. Frequency histogram of the selected antenna pairs (40 relays). 


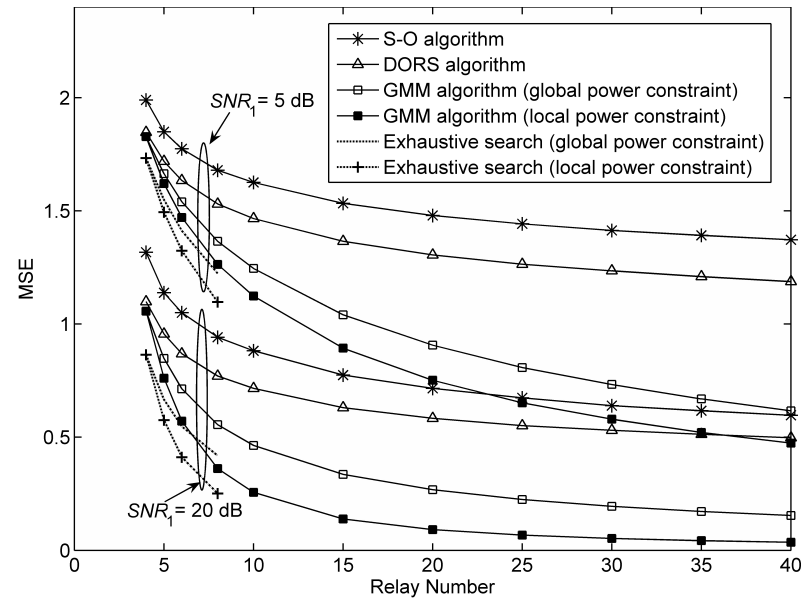

Fig. 3. MSE performance versus number of relay nodes $K$.

whereas the proposed GMM scheme tends to select more antennas $(M \leq l \leq K)$ until the MSE begins to increase. In each antenna selection loop, although the GMM scheme is more involved than the DORS and S-O algorithms, its implementation is feasible as explained earlier. Fig. 2 presents the frequency histogram of the selected antenna pairs for the GMM scheme for $K=40$. It is not surprising to find that less antenna pairs will be expected to participate the relaying when $S N R_{1}$ is smaller because the received signals at the relay nodes are more likely to vanish under the noise floor. However, we do observe that the GMM scheme will turn on more relay nodes than the DORS and S-O algorithms, thus making the performance comparison unfair due to the additional power gain. A simple way to separate the power gain from the enhancement offered by the GMM scheme is to pose a global power constraint on the selected $L$ forward antennas, i.e., instead of allocating $P_{\text {loc }}$ for each activated relay node, we dilute the relay power to $M P_{\text {loc }} / L$. Thereby, the total power at the activated relay nodes will be the same for the DORS, S-O and GMM schemes.

Fig. 3 shows the MSE performance versus relay number for the DORS, S-O, GMM and exhaustive search schemes. From Fig. 3, we find that the GMM scheme largely reduces the MSE compared to the DORS and S-O algorithms, with the gain being more conspicuous when the noise issue at the relay nodes becomes more serious (the upper set of curves, $S N R_{1}=5 \mathrm{~dB}$ ) and $K$ becomes larger. When comparing the curves for the GMM algorithm with and without global power constraint, we can draw the conclusion that the GMM algorithm without the power bonus has already reaped most of the performance gains. We also observe that the GMM scheme achieves the performance close to that by exhaustive search, which further confirms the superiority of the proposed scheme.

To illustrate how the MSE performance gains in Fig. 3 are interpreted into BER decrease, we plot the average BER curves in Fig. 4 with $S N R_{1}$ varying from $0 \mathrm{~dB}$ to $30 \mathrm{~dB}$. For all SNR cases, we deploy 15 relays. In addition, we assume that the Wiener filter is employed as the symbol detection filter, and the symbols are obtained from the QPSK constellation. As seen from Fig. 4, the proposed GMM scheme shows much

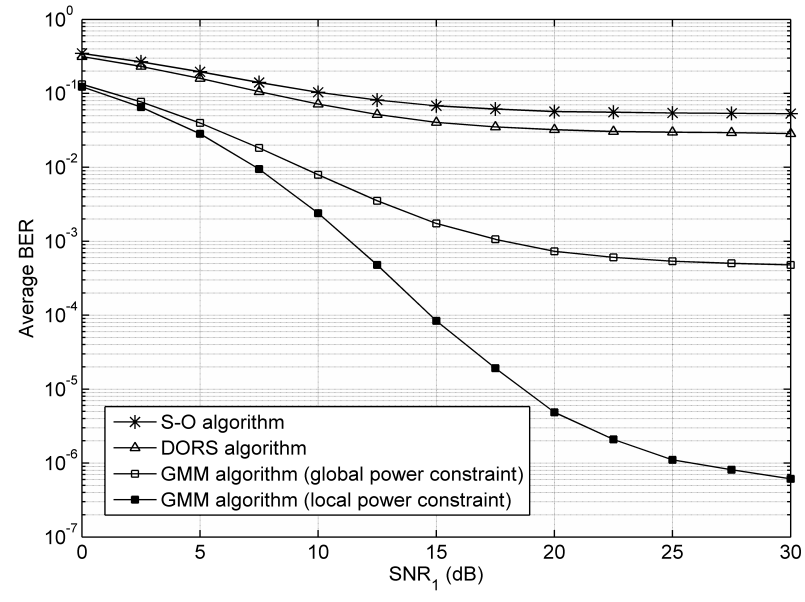

Fig. 4. BER performance versus $S N R_{1}(\mathrm{~dB})$.

steeper BER slope, indicating that more diversity is exploited in the system. With respect to the error floor caused by the limited power in the second hop channels, the proposed GMM scheme achieves considerable gains in orders.

\section{CONCLUSION}

In this paper, we proposed a greedy antenna selection algorithm to minimize the MSE resulted from selecting an additional relay antenna of an AF MIMO relay system. To reduce the complexity, the antenna selection process is carried out iteratively. Simulation results show that the proposed scheme exhibits much better performance than the existing schemes in terms of MSE and BER, and the gain is more pronounced in noisy channels.

\section{REFERENCES}

[1] B. Wang, J. Zhang, and A. Host-Madsen, "On the capacity of MIMO relay channels," IEEE Trans. Inf. Theory, vol. 51, no. 1, pp. 29-43, Jan. 2005.

[2] H. Bolcskei, R. Nabar, O. Oyman, and A. J. Paulraj, "Capacity scaling laws in MIMO relay networks," IEEE Trans. Wireless Commun., vol. 5, no. 6, pp. 1433-1444, Jun. 2006.

[3] W. Guan, H. W. Luo, and W. Chen, "Linear relaying scheme for MIMO relay system with QoS requirements," IEEE Signal Processing Letters, vol. 15 , pp. 697-700, 2008.

[4] C. B. Chae, T. Tang, R. W. Heath, S. Cho, "MIMO relaying with linear processing for multiuser transmission in fixed relay networks," IEEE Trans. Signal Processing, vol. 56, no. 2, pp.727-738, Feb. 2008.

[5] R. Pabst, B. H. Walke, D. C. Schultz, P. Herhold, H. Yanikomeroglu, S. Mukherjee, H. Viswanathan, M. Lott, W. Zirwas, M. Dohler, H Aghvami, D. D. Falconer, and G. P. Fettweis, "Relay-based deployment concepts for wireless and mobile broadband radio," IEEE Commun. Mag., vol. 42, no. 9, pp. 80-89, Sep. 2004.

[6] S. Chen, W. Wang, X. Zhang, and D. Zhao, "Performance of amplifyand-forward MIMO relay channels with transmit antenna selection and maximal-Ratio combining", in Proc. IEEE Wireless Communications and Networking Conf., Budapest, Hungary, Apr. 2009, pp. 1-6.

[7] S. W. Peters and R. W. Heath, "Nonregenerative MIMO relaying with optimal transmit antenna selection," IEEE Signal Processing Letters, vol. 15, pp. 421-424, 2008.

[8] W. Zhang, K. B. Letaief, "Opportunistic relaying for dual-hop wireless MIMO channels," in Proc. IEEE Global Telecommunications Conf. (GLOBECOM08), New Orleans, LO, USA, Nov. 2008, pp. 3985-3989.

[9] M. A. Torabi, J. F. Frigon, "Semi-orthogonal relay selection and beamforming for amplify-and-forward MIMO relay channels," in Proc. IEEE Wireless Communications and Networking Conf., Las Vegas, USA, Mar. 2008, pp. 48-53. 
[10] W. Guan, H.W. Luo, "Joint MMSE transceiver design in nonregenerative MIMO relay systems," IEEE Communications Letters, vol. 12, no. 7, pp. 517-519, 2008.

[11] S. Boyd, L. Vandenberghe, Convex Optimization. Cambridge, United Kingdom: Cambridge University Press, 2004. 